\title{
Numerical Identification of Solitary Wave-Like solutions in Boussinesq Equation as Inverse Problem
}

\author{
Tchavdar T. Marinov ${ }^{1}$, Brandon Bailey \\ Department of Natural Sciences, Southern University at New Orleans, 6400
}

\begin{abstract}
An algorithm to investigate numerically the solitary wave-like solutionsof Boussinesq differential equation is constructed. The bifurcation problem is re-formulated as an inverse problem for coefficient identification, introducing a newparameter. Such a way the nontrivial solution is separated from the trivial one.The Method of Variational Imbedding (MVI) is used for solving the inverse prob-lem. As illustration of the approach a Mathematica code with the finite differencescheme is prepared and the numerical results, including verification of the scheme,connection between the solution and the coefficients, and capability of Mathematica to paralelize the algorithm are presented.
\end{abstract}

\section{INTRODUCTION}

Almost two centuries ago John Scott Russell observed and chased on horseback (see forexample [5]) a solitary wave along a narrow channel and he gave this phenomenon the name the Wave of Translation. Later Boussinesq came up with a fundamental idea that the balance between the nonlinearity and dispersion is what makes the shape of the wave permanent [1]. Boussinesq's theory was the first to give a satisfactory and scientific explanation of the phenomenon of solitary waves discovered by Scott Russell. He foundalso the first analytical soliton like solution of sech-type

One faces two main challenges then solve numerically solitary wave equations. The first difficulty arises from the fact that the original problem is posed over an infinite interval. This means that any truncation of the computational domain will inevitablyentail dealing with numerical artifacts ensuing from the truncation of the region. The most successful techniques among the available from the literature make use of coordi-nate transformations or spectral expansion or both [2]. Yet, the truncation of the infinite interval with finite difference discretization is not the hardest hurdle and it gives satis-factory results when carefully implemented. The second, end sometimes harder difficulty,is that the existence of a solitary wave is the result of a bifurcation. Due to the nature of asymptotic boundary conditions for decay of the amplitude of the wave at infinity,the system under consideration always possesses a trivial solution. The above outline ddifficulties are much harder when the solutions have nonmomotone tails and when the intervals where they are well separated from zero, are large (see [3]). In many instances,the problem of finding the nontrivial amplitude of the bifurcating solution is akin to the problem of coefficient identification.

A new approach to creating effective algorithms for treating the bifurcation prob-lem connected with the localized solution was proposed in [7] obtaining numerically thesolitary-wave solutions of the Sixth-Order Generalized Boussinesq Equation, or 6GBE (see[4] for the derivation of that equation). The idea of this approach is to replace the original bifurcation problem with an inverse problem for coefficient identification. The unknown coefficient was identified by using the so-called Method of Variational Imbedding (MVI). The latter consists in replacing the original (inverse) problem by the Euler-Lagrange equations for minimization of a quadratic functional of the governing equations.

The paper is organized as follows. In Section 2 the problem is formulated. In Sec-tion 3 the problem is reformulated as an inverse problem for coefficient identification from overposed boundary data. The method of variational imbedding for solving this problem is applied in Section 4. The numerical scheme and algorithm is described in Section 5.Some numerical examples and results are given in Section 6.

\section{Problem formulation}

Consider the classical Boussinesq equation

$-\mathrm{u}_{\mathrm{tt}}+\gamma^{2} \mathrm{u}_{\mathrm{xx}}+\alpha\left(\mathrm{u}^{2}\right)_{\mathrm{xx}}-\mathrm{u}_{\mathrm{xxxx}=0}$,

\footnotetext{
${ }^{1}$ Corresponding Author: tmarinov@suno.edu
} 
Looking for nontrivial solution under boundary conditions.

$\mathrm{u} \rightarrow 0$ when $\mathrm{x} \rightarrow \pm \infty$.

Let us consider the stationary waves in the moving frame $\xi=\mathrm{x}-$ ct. After double integration with respect to $\xi$ and taking into account the localized character of the investigated solutions, one obtains the following nonlinear ordinary differential equation

$\mathrm{L}(\mathrm{u})=\lambda \mathrm{u}+\alpha \mathrm{u}^{2}+\mathrm{u}_{\xi \xi}=0, \lambda \equiv \gamma^{2}-\mathrm{c}^{2}$,

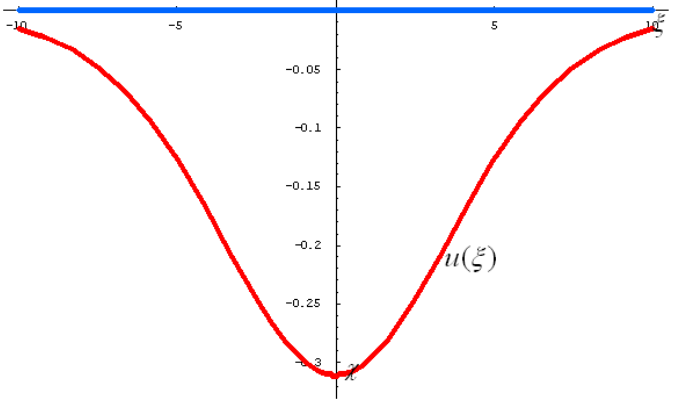

a)

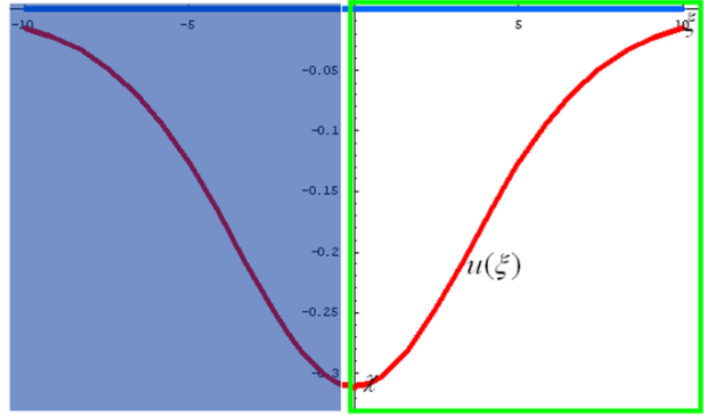

b)

We are looking for solutions of the Eq. (3) with $\mathrm{u} \rightarrow 0$ when $\xi \rightarrow \infty$. Then $\mathrm{u}^{2}<<\mathrm{u}$ in the tails and the linearized version of (3) coincides with its linear part. It possesses harmonic solutions of the type $\mathrm{e}^{\mathrm{k} \xi}$. The corresponding dispersion relation reads

$\mathrm{k}^{2}+\lambda=0 \quad \Rightarrow \quad \mathrm{k}= \pm \sqrt{ }-\lambda$

Equation (4) shows that the natural classification of stationary solutions should be based on the spatial asymptotic behavior of the tails. First: a stationary solution can be subsonic $(\lambda>0)$ or supersonic $(\lambda<0)$; Second: the asymptotic tails of the localized wave can be either monotonic or purely oscillatory depending on whether $\mathrm{k}$ is real or complex. A similar problem usually arises when one tries to identify numerically a solitary-wave-like solution.

\section{INTRODUCING UNKNOWN COEFFICIENT}

Since a trivial solution $u \equiv 0$ exists, the problem (3), (2) is ill-posed in sense of Hadamard because there are two solutions for given boundary conditions. In addition, the trivialsolution is a very strong attractor. For this reason, it is difficult to construct a numericalscheme for solving this problem.

Let $u(x) 6 \equiv 0$ be a solution of the equation (3) under boundary conditions (2). Obvi-ously, the function $u(-x)$ is also a solution, i.e. the solution is an even function (satisfyingthe condition $u(x)=u(-x)$ ). This fact allows us to consider the problem on the half-line(see Figure 1 ). Then the boundary conditions for $\mathrm{x}=0$ reads:

$\mathrm{u}(0)=\chi$,

$\mathrm{u}^{\prime}(0)=0$, 


$$
\mathcal{A}(y, \chi)=y^{\prime \prime}(x)+\alpha \chi y^{2}(x)+\lambda y(x)=0,
$$

under boundary conditions

$\mathrm{y}(0)=1$,

$\mathrm{y}^{\prime}(0)=0$,

$\mathrm{y}^{\prime}(\mathrm{x}) \rightarrow 0$ when $\mathrm{x} \rightarrow \infty$.

Thus the difficulties connected with the unknown constant $\chi$ in the boundary condition(5) are circumvented. Yet, it is a problem of unknown coefficient $\chi$ from over-posedboundary data. Under certain natural conditions it is possible to find a constant $\chi$ such that the equation (8) has a solution $\mathrm{y}(\mathrm{x})$ and this solution also satisfies the boundary conditions (9)-(11). In such a case we say that the pair $(\mathrm{y}, \chi)$ constitute a solution tothe problem (8)-(11).

\section{VARIATIONAL IMBEDDING}

In [7] a special numerical technique has been developed for identification symmetric of solitary wave solutions of Boussinesq and Korteweg-de Vries equations was proposed.The original ill-posed problem is transferred into a problem of the unknown coefficient from over-posed boundary data in which the trivial solution is excluded. The Method of Variational Imbedding (MVI) is used for solving the inverse problem (see for example[8, 9], and [6] in case of elliptic PDE's).

Following the method proposed in [7], we replace the original problem by the problem of minimization of the functional

$$
\mathcal{I}(y, \chi)=\int_{0}^{\infty}[\mathcal{A}(y, \chi)]^{2} \mathrm{~d} x=\int_{0}^{\infty}\left[y^{\prime \prime}(x)+\alpha \chi y^{2}(x)+\lambda y(x)\right]^{2} \mathrm{~d} x \rightarrow \min ,
$$

where y must satisfy the conditions (9)-(11) and $\chi \neq 0$ is an unknown constant. The functional $\mathrm{I}$ is a quadratic and homogeneous function of the $\mathrm{A}(\mathrm{y}, \chi)$ and hence it attains its minimum if and only if $\mathrm{A}(\mathrm{y}, \chi) \equiv 0$. In this sense there is a one-to-one correspondence between the solution of the original problem (8)-(11) and the minimization problem (12), (10)-(11).

Clearly, the Euler(-Lagrange) equation for this functional possesses a cubic nonlinear-ity with respect to the function y. This means that for the numerical solution one has to linearize the said equation in order to solve it numerically. Alternatively, one can linearize the integrand in (12), considering the function y as known (say, from the previous itera-tion) when it appears on second degree, i.e., $\mathrm{y}^{2}(\mathrm{x})=\mathrm{q}(\mathrm{x})$. Following the second approach for the linearization, consider the problem of minimization of the following functional.

$$
\mathcal{I}(y, \chi)=\int_{0}^{\infty}[F(x)]^{2} \mathrm{~d} x \rightarrow \min
$$

Where

$$
F(x) \equiv y^{\prime \prime}(x)+\alpha \chi q(x)+\lambda y(x)
$$

is the residual of the given equation. The advantage of this linearization is that the matrix of the linear system is the same during the calculation process and we do not need to invert it multiple times.

\subsection{Imbedded Boundary-Value Problem}

Necessary conditions for minimization of (13) are the Euler-Lagrange equations for thefunction $\mathrm{y}(\mathrm{x})$ and coefficient $\chi$. The equation for y reads 


$$
\left(\frac{\mathrm{d}^{2}}{\mathrm{~d} x^{2}}+\lambda\right) F(x)=y^{(4)}+2 \lambda y^{\prime \prime}+\lambda^{2} y+\alpha \chi\left(q^{\prime \prime}+q\right)=0
$$

This equation is of fourth order and its solution can satisfy the boundary conditions (9)-(11), i.e., the boundary value problem for equation (15) under boundary conditions(9)-(11) is not over determined.

The problem is coupled with the equation for $\chi$. We rewrite the functional (12) in the form

$$
\mathcal{I}=\chi^{2} \alpha^{2} \int_{0}^{\infty} y^{4} \mathrm{~d} x+2 \chi \alpha \int_{0}^{\infty} y^{2}\left(y^{\prime \prime}+\lambda y\right) \mathrm{d} x+\int_{0}^{\infty}\left(y^{\prime \prime}+\lambda y\right)^{2} \mathrm{~d} x
$$

and after some manipulations the equation for $\chi$ adopts the form

$$
\chi=-\frac{\int_{0}^{\infty} y^{2}\left(y^{\prime \prime}+\lambda y\right) \mathrm{d} x}{\alpha \int_{0}^{\infty} y^{4} \mathrm{~d} x}
$$

\section{FINITE DIFFERENCE SCHEME}

The mesh (see Fig. 2) is regular and allows to approximate all operators with standard central differences.

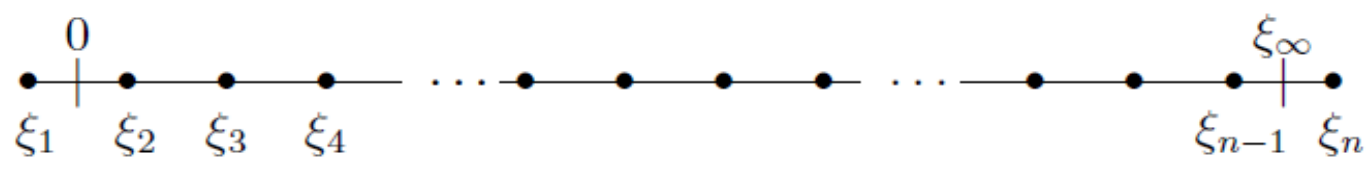

Fig2: The mesh

\subsection{Grid Pattern and Approximations}

The grid spacing is

$h \equiv \frac{\xi_{\infty}}{n-2}$,

where $\mathrm{n}$ is the total number of grid points and $\xi_{\infty}$ is a sufficiently large number, called'numerical infinity'. Then the gird points are defined as follows

$\xi_{\mathrm{i}}=(\mathrm{i}-1.5) \mathrm{h}$ for $\mathrm{i}=1, \ldots, \mathrm{n}$.

It is an important fact that the point $\xi=0$ is the mid-point $\xi_{1 \frac{1}{2}}$

$y_{i}=y\left(\xi_{i}\right), \quad$ for $\quad i=1, \ldots, n$

I employ symmetric central differences for approximating the differential operators as follows

$y^{(4)}\left(\xi_{i}\right)=\Lambda_{\xi \xi \xi \xi} y_{i}+O\left(h^{2}\right)=\frac{1}{h^{4}}\left(y_{i-2}-4 y_{i-1}+6 y_{i}-4 y_{i+1}+y_{i+2}\right)+O\left(h^{2}\right)$

for $\mathrm{i}=3, \ldots, \mathrm{n}-2$, and

$y^{\prime \prime}\left(\xi_{i}\right)=\Lambda_{\xi \xi} y_{i}+O\left(h^{2}\right)=\frac{1}{h^{2}}\left(y_{i-1}-2 y_{i}+y_{i+1}\right)+O\left(h^{2}\right)$

for $\mathrm{i}=2, \ldots, \mathrm{n}-1$. 
The grid allows approximations of second order the boundary conditions as well

$$
\begin{aligned}
& y(0)=1 \quad \Longrightarrow y_{1}+y_{2}=2, \\
& y^{\prime}(0)=0 \quad \Longrightarrow y_{2}-y_{1}=0, \\
& y(\xi) \rightarrow 0, \quad \xi \rightarrow \infty \quad \Longrightarrow y_{n}+y_{n-1}=0, \\
& y^{\prime}(\xi) \rightarrow 0, \quad \xi \rightarrow \infty \quad \Longrightarrow y_{n}-y_{n-1}=0
\end{aligned}
$$

\subsection{Estimation of $\chi$}

We approximate the integrals in equation (17) for $\chi$ using so called 'extended midpoint rule'

$$
\chi=-\frac{\sum_{k=2}^{n-1} y_{k}^{2}\left(\Lambda_{\xi \xi} y_{k}-4 y_{k}\right)}{\alpha \sum_{k=2}^{n-1} y_{k}^{4}}+O\left(h^{2}\right)
$$

Here, the error term is again of the second order.

\subsection{Algorithm}

- The forth-order boundary value problem (16), (9)-(11) is solved for the function ywith given $\chi$ and $\mathrm{q}$.

- If the deviation of the newly calculated y from y from the previous iteration is smaller than $\varepsilon$ then we proceed to (III), otherwise q is replaced by $\mathrm{y}^{2}$ and then go to (I);

- With the newly computed $y$, the coefficient $\chi$ is evaluated from (26). If

$\left|\chi^{\text {new }}-\chi^{\text {old }}\right|<\varepsilon$,

then the calculations are terminated. Otherwise the index of iterations is stepped up $n:=n+1$ and the algorithm is returned to step (I).

\section{NUMERICAL SOLUTION WITH MATHEMATICA}

The problem (29), (2) posses an analytic solution

$$
u(\xi)=-\frac{3 \lambda}{2 \alpha} \operatorname{sech}^{2}\left( \pm \sqrt{\frac{-\lambda}{4} \xi}\right),
$$

under the condition $\lambda<0$.

If $\alpha=6$ and $\lambda=-4$, the equation (3) takes the form

$$
\mathcal{L}(u)=u^{\prime \prime}(\xi)+6 u^{2}(\xi)-4 u(\xi)=0,
$$

and the problem (29), (2) has an analytic solution

$\mathrm{u}^{\mathrm{an}}=\mathrm{u}(\xi)=\operatorname{sech}^{2}(\xi)$,

which can be used to validate the numerical schemes.

For numerical realization of the algorithm we are using Mathematica. 


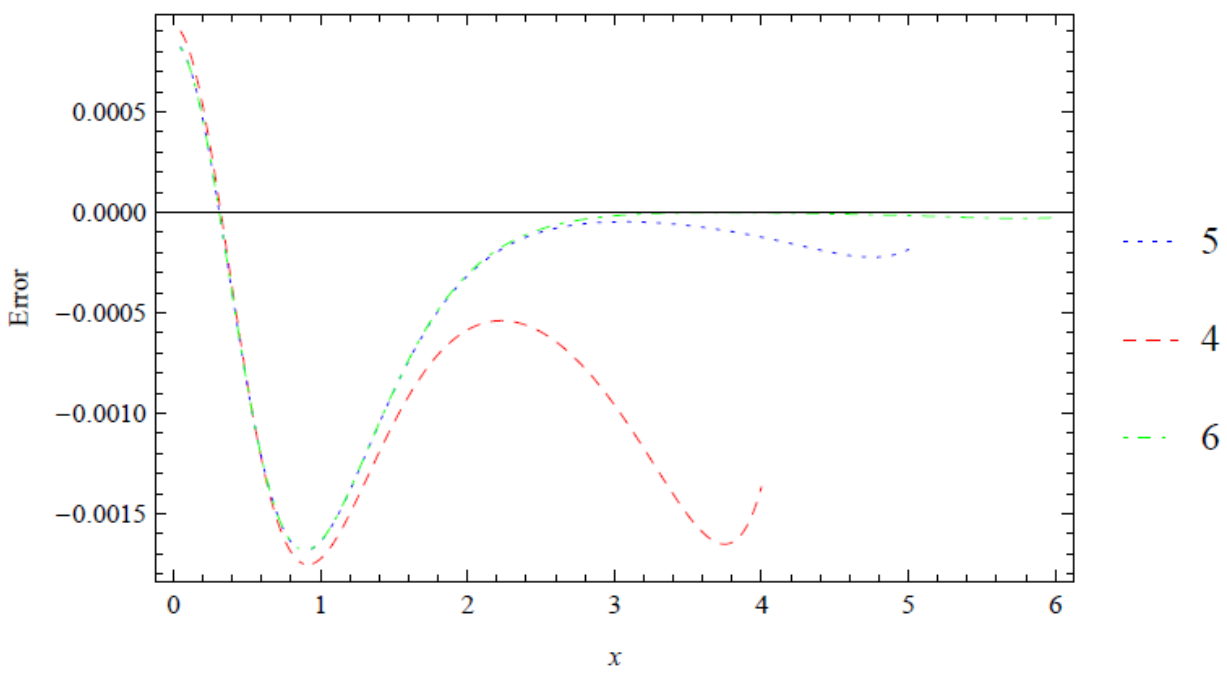

Fig3. Error distribution for $\xi_{\infty}=4$ (dashed red line), $\xi_{\infty}=5$ (dotted blue line), $\xi_{\infty}=6$ (dash-dotted green line).

\subsection{Numerical Tests for $\xi_{\infty}$}

The first test is connected with'numerical infinity ' $\xi_{\infty}$. At Figure 3 the distribution of the numerical error for three different values of $\xi_{\infty}{ }^{\prime} \xi_{\infty}=4,5,6$,) are shown. Wekeep the step $\mathrm{h}=0.1$ during all calculations. The difference between numerical and exact solutions is not significant for $\xi_{\infty}=6$ and, therefore, we use $\xi_{\infty}=12$ for the next numerical experiments.

\subsection{Numerical Test of Convergence}

Since the problem has an exact solution (30) we conduct some experiments in order to verify the dizcretization error term.

The discretization error term is $\mathrm{O}\left(\mathrm{h}^{2}\right)$, and the total error is $\mathrm{O}\left(\mathrm{h}^{2}\right)$. The numerical results demonstrate clearly these error orders. The values of the identified coefficient $\chi$ and $\left\|\mathrm{u}^{\text {num }}-\mathrm{u}^{\mathrm{an}}\right\|$ calculated with five different steps are given in Table 1. The rates of convergence, calculated as

$$
\operatorname{rate}(\chi)=\log _{2}\left|\frac{\chi_{2 h}-\chi_{\text {an. }}}{\chi_{h}-\chi_{\text {an. }}}\right| \quad \text { and } \quad \operatorname{rate}(u)=\log _{2} \frac{\left\|u_{2 h}^{n u m}-u^{a n}\right\|}{\left\|u_{h}^{n u m}-u^{a n}\right\|}
$$

are shown also in Table 1.

The point-wise distribution of the error for four different calculations with $\xi_{\infty}=12$ and $h=1 / 10 ; 1 / 20 / 1 / 40 ; 1 / 80$, is shown at Figure4.The experiments clearly confirm the theoretical $\mathrm{O}\left(\mathrm{h}^{2}\right)$ convergence

Table1. Obtained values of the amplitude $\chi$ of the peak, $\left\|y^{\text {num }}-y^{\text {an }}\right\|$, and the rate of convergence for five different values of the mesh spacing

\begin{tabular}{|c|c|c|c|c|}
\hline $\mathrm{h}$ & $\chi$ & rate of convergence & $\left\|\mathrm{u}^{\text {num }}-\mathrm{u}^{\text {an }}\right\|$ & rate of convergence \\
\hline analytical & 1.000000 & - & - & - \\
\hline $1 / 5$ & 0.993152 & - & 0.0063308500 & - \\
\hline $1 / 10$ & 0.998327 & 2.03325 & 0.0015391100 & 2.04030 \\
\hline $1 / 20$ & 0.999588 & 2.02172 & 0.0003865510 & 1.99337 \\
\hline $1 / 40$ & 0.999902 & 2.07179 & 0.0001010250 & 1.93595 \\
\hline $1 / 80$ & 0.999976 & 2.02975 & 0.0000260587 & 1.95488 \\
\hline
\end{tabular}




\subsection{Shape of the solution for different values of $\alpha$.}

The shapes of the solution for four different values of the coefficient $\lambda=-4 ;-5 ;-6 ;-7$, are given in Figure 5 . During the calculations we keep the parameters as $h=1 / 100, \alpha=6, \xi_{\infty}=12$.

\section{ACKNOWLEDGEMENT}

The work of BB was supported by the NSF Grant DMS: 1214359

\section{REFERENCES}

[1] Boussinesq, J. [1871] "Theorie de l'intumescence liquide appelee onde solitaire oude translation se propageant dans un canal rectangulare",Compte Rendus AcadSci. 72, 755-759.

[2] J. P. Boyd, Chebishev and Fourier Spectral Methods, Dover, New York, 2001, secondEdition.

[3] C.I. Christov, T.Marinov, R.S. Marinova Identification of solitary-wave solutions asan inverse problem: application to shapes with oscillatory tails Mathematics andComputers in Simulation, 80(1), 56-65, (2009).

[4] C. I. Christov, G. A. Maugin, M. G. Velarde, On the well-posed Boussinesq Paradigm with purely spatial higher-order derivatives, Phys. Rev. E 54 (1996) 3621-3638.

[5] Russell J. Scott [1844] "Report on waves".14th Meeting of the British Association Report, (York).

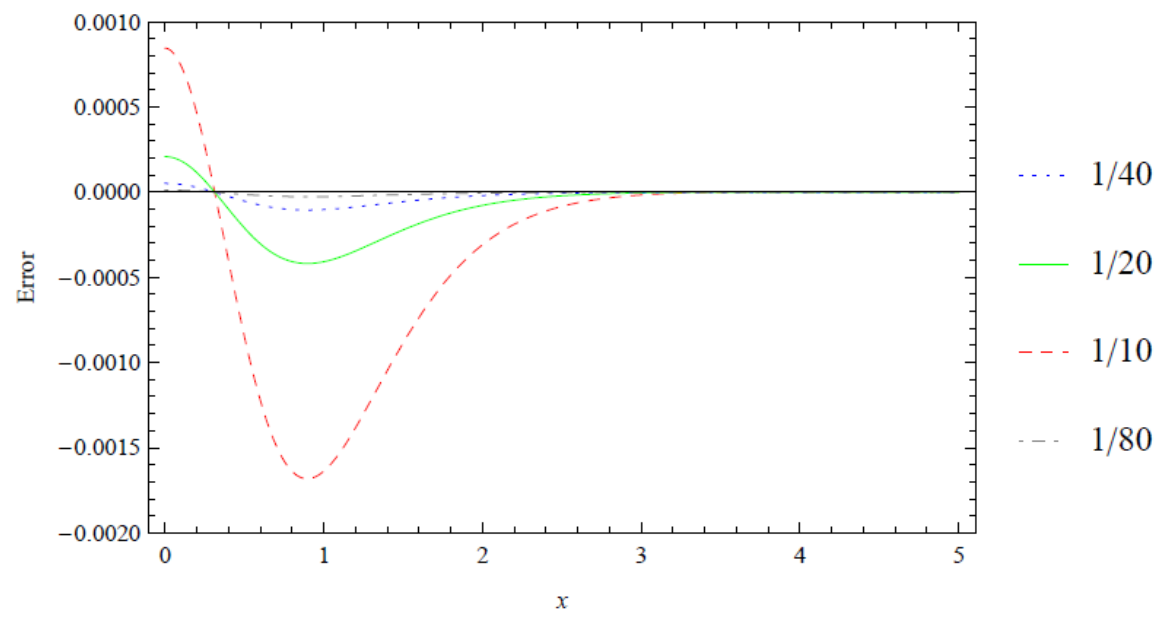

Fig4. Error distribution for $\xi_{\infty}=12$ for four different space steps $h=1 / 10$ (dashed red line), $h=1 / 20$ (solid green line), $\mathrm{h}=1 / 40$ (dotted blue line), $\mathrm{h}=1 / 80$ (dot-dashed gray line).

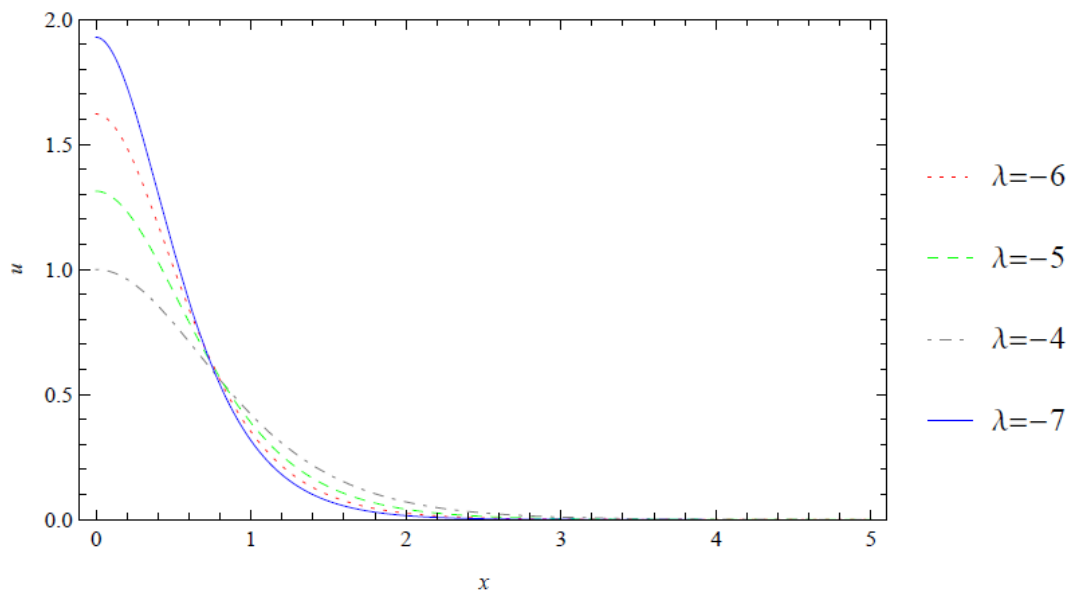

Fig5. The shape of the solution for four different values of $\lambda=-4$, (dot-dashed grayline), $\lambda=-5$, (dashed greenline), $\lambda=-6$, (solid blue line), $\lambda=-7$, (solid gray line). 
American Research Journal of Mathematics, Volume 1, Issue 1, February 2015

ISSN 2378-704X

[6] T.T. Marinov, R.S. Marinova, C.I. Christov, Coefficient identification in elliptic par-tial diff erential equation, in: J.W.I. Lirkov, Sv. Margenov (Eds.),Lecture Notes in Computer Science, vol. 3743, Springer, 2006, pp. 372379.

[7] T.Marinov, C.I.Christov, R.S.Marinova, Novel Numerical Approach to Solitary-WaveSolutions Identification of Boussinesq and Korteweg-de Vries Equations. Int. Journal of Bifurcation and Chaos. Vol. 15, No. 2, 2005.

[8] T.T. Marinov, R.S. Marinova, Coefficient identification in EulerBernoulli equation from overposed data, J. Comput. Appl. Math. 235 (2) (2010) 450459.

[9] T.T. Marinov, A. Vatsala, Inverse problem for coefficient identification in Euler- BernoulliEquation, Comput. Math. Appl. 56 (2) (2008) 400410. 